\title{
Un nuevo modelo semidefinido de análisis dinámico de estructuras
}

\author{
J.L. Urrutia-Galicia \\ Coordinación de Mecánica Aplicada \\ Instituto de Ingeniería, UNAM \\ E-mail:jurg@pumas.iingen.unam.mx
}

(recibido: octubre de 2001; aceptado: diciembre de 2002)

\begin{abstract}
Resumen
En este artículo se presenta un modelo semidefinido en el espacio complejo para el análisis dinámico de estructuras. En esta ocasión se analizarán detalladamente los mecanismos de vibración en función de un semiespacio móvil (el apoyo) con masa $m_{0}$, un sistema principal con masa $m_{1}$ y otra masa $m_{2}$. Las tres masas están unidas mediante resortes $\left(k_{1}\right.$ y $\left.k_{2}\right)$ y amortiguadores $\left(\xi_{1}\right.$ y $\left.\xi_{2}\right)$ y en el análisis final se considera que la masa $m_{0}>>>>m_{1}+m_{2}$. La solución en el espacio complejo permite hacer un análisis claro de los ángulos de fase de las tres masas, vibrando a diferentes frecuencias de excitación $\omega$, lo cual permite observar las masas $m_{1}$ y $m_{2}$ vibrando e incluyendo la influencia del semiespacio $m_{0}$, es decir, se analiza simultáneamente el problema de la transmisibilidad de vibraciones. Se mostrará que el nuevo modelo es útil no sólo en problemas clásicos de excitación en las masas de la estructura principal, sino también en problemas donde la excitación llega por la frontera o en aquellos donde la estructura no tiene una posición fija, como es el caso de los robots.
\end{abstract}

Descriptores: modelo complejo semidefinido, dinámica general, transmisibilidad de vibraciones, movimiento en la base, fasores, ángulos de fase, módulo de un número complejo, robots.

\section{Abstract}

The paper presents a semidefinite model in the complex space for the dynamic analysis of structures. This time a careful analysis of the vibration mechanisms will be presented as a function of the mobile halfspace with mass $m_{0}$, and a main system with two masses $m_{1}$ and $m_{2}$. The three masses are joined by arbitrary springs and dampers and the analysis considers that $m_{0}>>>m_{1}+m_{2}$. The complex solution allows for a detailed view of the phase angles of the three masses conforming the complete system at different excitation frequencies that in turn let observe the two masses $m_{1}$ y $m_{2}$ and the influence of the half space represented by the mass $m_{0}$ so we can see the problem of transmisibility. The new model can be applied to classical structures where the main structure is excited or in cases when: the excitation arrives by the boundary or the structure has a mobile nature like that of robots.

Keywords: semidefinite complex model, general dynamics, transmisibility of vibrations, base motion, phasors, phase angle, modulus of a complex number, robots. 


\section{Introducción}

La filosofía general de diseño de estructuras civiles se hace con modelos cuyas fronteras están soportadas de una forma que se garantice, en primera instancia, la estabilidad de una viga, un edificio o de cualquier otra estructura. La fijación de las fronteras se expresa algebraicamente imponiendo la condición de que el modelo matemático sea positivo definido. Si la excitación - excitaciones (desplazamientos, fuerzas o aceleraciones) se aplican en puntos hacia el interior de la frontera y en cualquier punto de la estructura, entonces los modelos positivo definidos, vigas placas o cascarones, nos darán soluciones admisibles y físicamente explicables en la mayoría de los casos. Sin embargo, en ciertos problemas nos conducen a modelos diferentes, difíciles, y por lo tanto a conclusiones inciertas (Bettin et al., 2000). Por lo anterior, una filosofía invariante (Urrutia, 1998) y técnicas diferentes serán discutidas y aplicadas a un modelo lineal ( $\sin$ daño o con deterioro mínimo) que incluya una estructura principal (con dos grados de libertad) y un semiespacio móvil absorbente, acoplado dinámicamente mediante resortes.

La falta de claridad en los modelos ha generado mucha controversia. En este trabajo se presenta un modelo constituido (para el modelo más simple) por un elemento de apoyo móvil con masa $m_{0}$ finita, un sistema principal con dos masas $m_{1} y$ $m_{2}$ conectados entre sí por resortes y amortiguadores. En esta primera presentación del modelo la masa $m_{0}$ es muchas veces mayor que la suma de $m_{1}+m_{2}$. Este modelo manejará tres formas modales, a saber: el primer modo o modo de cuerpo rígido, con las tres masas vibrando juntas y en fase, así como los dos modos restantes ya conocidos (más utilizados), que incluirán la interacción entre el semiespacio $m_{0}$ en balance dinámico con las otras dos masas $m_{1}$ y $m_{2}$.

\section{Modelo matemático}

La figura 1 es un esquema de un sistema semidefinido, constituido por las masas $m_{0}, m_{1}$ y $m_{2}$ que están acopladas físicamente por las rigideces $k_{1}$ y $k_{2}$ y los amortiguadores $c_{1}$ y $c_{2}$.
La masa $m_{0}$ puede representar el apoyo del sistema principal $m_{1}$ y $m_{2}$ cuando el sistema a controlar está empotrado, o la masa del apoyo, cuando el sistema a controlar se localiza, por ejemplo, a bordo de un helicóptero o de algún vehículo. Claro que el modelo de la figura 1 se convierte en un sistema definido de dos grados de libertad si se supone que la masa $m_{0}$ es de magnitud muy grande o infinita.

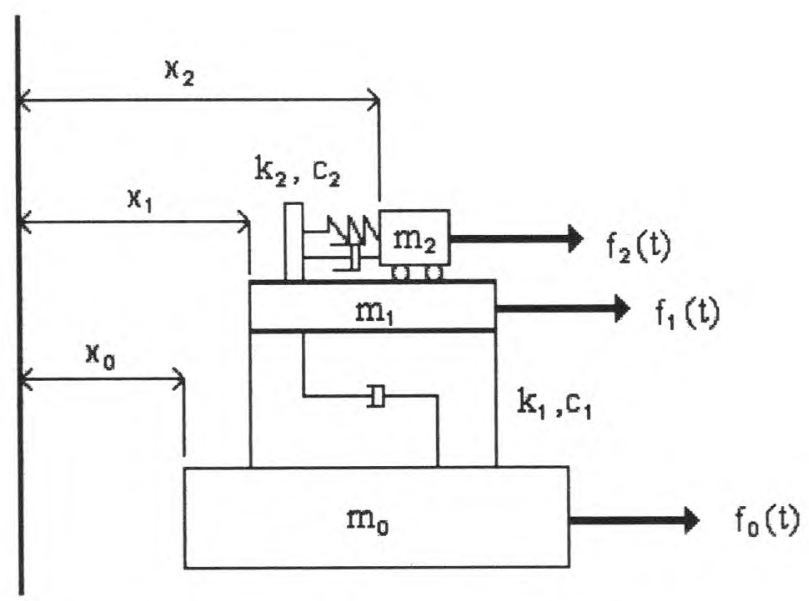

Figura 1. Sistema semidefinido

La masa $m_{0}$ se llamará "semiespacio". La masas $m_{1}$ y $m_{2}$, sus elementos de acoplamiento, la rigidez $k_{i}$ y el a mortiguador viscoso $c_{i}$, serán "el sistema principal". El arreglo completo de la figura 1 será "el sistema completo" o "el sistema". Las fuerzas $f_{a r} f_{1} y f_{2}$ son funciones del tiempo y representan a las excitaciones que pueden ser aplicadas sobre el sistema.

\section{Ecuaciones de movimiento}

Las ecuaciones de movimiento del sistema están descritas por:

$$
\begin{gathered}
{\left[\begin{array}{ccc}
m_{0} & 0 & 0 \\
0 & m_{1} & 0 \\
0 & 0 & m_{2}
\end{array}\right]\left[\begin{array}{l}
\ddot{x}_{0} \\
\ddot{x}_{1} \\
\ddot{x}_{2}
\end{array}\right]+\left[\begin{array}{ccc}
c_{1} & -c_{1} & 0 \\
-c_{1} & c_{1}+c_{2} & -c_{2} \\
0 & -c_{2} & c_{2}
\end{array}\right]} \\
{\left[\begin{array}{ccc}
k_{1} & -k_{1} & 0 \\
-k_{1} & k_{1}+k_{2} & -k_{2} \\
0 & -k_{2} & k_{2}
\end{array}\right]\left[\begin{array}{l}
x_{0} \\
x_{1} \\
x_{2}
\end{array}\right]=\left[\begin{array}{l}
f_{0} \\
f_{1} \\
f_{2}
\end{array}\right]}
\end{gathered}
$$


si se supone una excitación armónica del tipo

$$
f_{j}(t)=F_{j} e^{i \omega t}, j=0,1,2, \quad i=\sqrt{-1}
$$

la respuesta cinemáticamente compatible de desplazamiento absoluto del sistema es de la siguiente forma

$$
x_{j}(t)=X_{j} e^{i \omega t}, j=0,1,2
$$

por lo que las derivadas con respecto al tiem po son

$$
\left[\begin{array}{l}
\dot{x}_{0} \\
\dot{x}_{1} \\
\dot{x}_{2}
\end{array}\right]=\left[\begin{array}{l}
i \omega X_{0} e^{i \omega t} \\
i \omega X_{1} e^{i \omega t} \\
i \omega X_{2} e^{i \omega t}
\end{array}\right] \quad\left[\begin{array}{l}
\ddot{x}_{0} \\
\ddot{x}_{1} \\
\ddot{x}_{2}
\end{array}\right]=\left[\begin{array}{l}
-\omega^{2} X_{0} e^{i \omega t} \\
-\omega^{2} X_{1} e^{i \omega t} \\
-\omega^{2} X_{2} e^{i \omega t}
\end{array}\right]
$$

Al sustituir estas últimas expresiones en (1) se obtiene donde se introdujeron las siguientes definiciones y parámetros adimensionales

$$
\begin{gathered}
\omega_{1}=\sqrt{\frac{k_{1}}{m_{1}}} \quad \omega_{2}=\sqrt{\frac{k_{2}}{m_{2}}} \quad \zeta_{1}=\frac{c_{1}}{2 m_{1} \omega_{1}} \quad \zeta_{2}=\frac{c_{2}}{2 m_{2} \omega_{2}} \\
\alpha=\frac{m_{1}}{m_{0}} \quad \beta=\frac{m_{2}}{m_{1}} \quad \delta=\frac{\omega_{2}}{\omega_{1}} \quad \gamma=\frac{\omega}{\omega_{1}}
\end{gathered}
$$

con estas definiciones se resuelve la ecuación (6) y se obtienen los siguientes resultados adimensionales que sólo dependen de $\alpha, \beta, \delta$ y $\gamma$ dependiente de la frecuencia de excitación $\omega$ así como de los amortiguamientos $\xi_{1}$ y $\xi_{2}$ y de las cargas $F_{i}$ con $i=0,1$ y 2 .

$$
X_{0}=\frac{F_{0}\left(Z_{22} Z_{33}-Z_{23}{ }^{2}\right)-F_{1} Z_{12} Z_{33}+F_{2} Z_{12} Z_{23}}{\left[Z_{11} Z_{22} Z_{33}-Z_{11}\left(Z_{23}\right)^{2}-Z_{33}\left(Z_{12}\right)^{2}\right]^{*} k_{1}}
$$

$\left[\begin{array}{ccc}-\omega^{2} \cdot m_{0}+i \cdot \omega \cdot c_{1}+k_{1} & -i \cdot \omega \cdot c_{1}-k_{1} & 0 \\ -i \cdot \omega \cdot c_{1}-k_{1} & -\omega^{2} \cdot m_{1}+i \cdot \omega \cdot\left(c_{1}+c_{2}\right)+k_{1}+k_{2} & -i \cdot \omega \cdot c_{2}-k_{2} \\ 0 & -i \cdot \omega \cdot c_{2}-k_{1} & -\omega^{2} \cdot m_{2}+i \cdot \omega \cdot c_{2}+k_{2}\end{array}\right]\left[\begin{array}{l}X_{0} \\ X_{1} \\ X_{2}\end{array}\right]=\left[\begin{array}{l}F_{0} \\ F_{1} \\ F_{2}\end{array}\right]$

La matriz cuadrada del lado izquierdo es la llamada matriz de impedancia dinámica $Z_{i j}$ (Thompson, 1972), (Tse et al., 1978) y esto permite rescribir la ecuación (5) de la siguiente manera $\left(\operatorname{con} F_{0}, F_{1}\right.$ y $F_{2} \neq 0$ )

$$
\left[\begin{array}{ccc}
Z_{11} & Z_{12} & 0 \\
Z_{12} & Z_{22} & Z_{23} \\
0 & Z_{23} & Z_{33}
\end{array}\right]\left[\begin{array}{l}
X_{0} \\
X_{1} \\
X_{2}
\end{array}\right]=\left[\begin{array}{l}
F_{0} \\
F_{1} \\
F_{2}
\end{array}\right]
$$

en donde los elementos $Z_{i j}$ se rescriben de la siguiente manera

$$
\begin{array}{cc}
Z_{11}=\left\lfloor\left(1+i 2 \gamma \zeta_{1}\right)-\frac{\gamma^{2}}{\alpha}\right] & Z_{22}=\left\lfloor 1+\beta \delta^{2}-\gamma^{2}+i 2 \gamma\left(\zeta_{1}+\beta \delta \zeta_{2}\right)\right\rfloor \\
Z_{12}=-\left(1+i 2 \gamma \zeta_{1}\right) & Z_{23}=-\beta \delta\left(\delta+i 2 \gamma \zeta_{2}\right) \\
Z_{13}=0 & Z_{33}=\beta\left(\delta^{2}-\gamma_{2}+i 2 \gamma \delta \zeta_{2}\right)
\end{array}
$$

$$
X_{1}=\frac{F_{1} Z_{11} Z_{33}-F_{2} Z_{11} Z_{23}-F_{0} Z_{12} Z_{33}}{\left[Z_{11} Z_{22} Z_{33}-Z_{11}\left(Z_{23}\right)^{2}-Z_{33}\left(Z_{12}\right)^{2}\right]^{*} k_{1}}
$$

$$
X_{2}=\frac{F_{2}\left(Z_{11} Z_{22}-Z_{12}^{2}\right)-F_{1} Z_{11} Z_{23}+F_{0} Z_{12} Z_{23}}{\left[Z_{11} Z_{22} Z_{33}-Z_{11}\left(Z_{23}\right)^{2}-Z_{33}\left(Z_{12}\right)^{2}\right]^{*} k_{1}}
$$

Las fórmulas (9), (10) y (11) se introdujeron en el programa "Mathcad 7.0 Professional edition" y los análisis correspondientes se efectúan en forma rápida y con soluciones convergentes. El sistema común de dos grados de libertad se obtiene de esta presentación cuando $\alpha=m_{1} / m_{0} \rightarrow 0$. En los diversos análisis que se presentan se toma $\alpha=0.000001$. La variables $\beta=m_{2} / m_{1} y \delta=\omega 2 / \omega 1$ se seleccionan de acuerdo a diferentes criterios de análisis. Los amortiguamientos $\zeta_{1}$ y $\zeta_{2}$ se eligen según la estructura que se analiza. Con estos parámetros definidos se varía el último parámetro $\gamma=\omega / \omega 1$ desde aproximadamente 0.1 hasta 2.0 para observar la respuesta de toda la estructura 
(función de transferencia) y decidir si las vibraciones son o no adecuadas.

\section{Análisis de una estructura clásica de dos grados de libertad}

Ejemplo 1

Se tiene un sistema elástico constituido por un semiespacio, una masa principal $m_{1}$ y una segunda masa $m_{2}$. El semiespacio es suficientemente grande cuando $\alpha=m_{1} / m_{0}$ $=0.000001$ y por tanto el problema se reduce en el límite a un sistema de dos grados de libertad con una masa pequeña $m_{1}=4$ y una masa $m_{2}=4$, simulando así un sistema $m_{1}, m_{2}$ a poyado en el semiespacio $m_{0}$. Con esto, el valor $\beta=m_{2} / m_{1}=1.0$ queda ya definido. Los otros parámetros elegidos son: $\zeta_{1}=0.10, \zeta_{2}=0.15$ y el primer valor de $\delta=\omega 2 / \omega 1=0.0001$. El valor de $\delta=0.0001$ prácticamente desliga al absorbedor del resto del sistema y reduce el problema a un sistema de un grado de libertad. Hasta este momento sólo nos queda como parámetro libre $\gamma=\omega / \omega 1$, al cual asignamos valores desde 0.1 hasta 2.0 obteniendo las siguientes funciones de
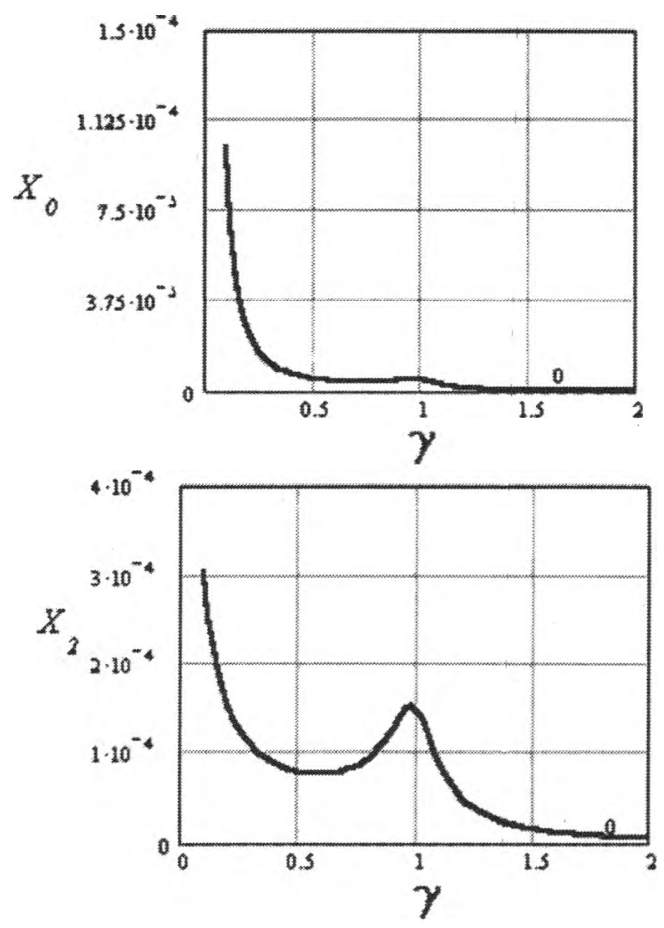

transferencia $X_{0}, X_{1}$ y $X_{2}$ para las tres masas $m_{0}$, $m_{1}$ y $m_{2}$. Se supone que el sistema está sólo bajo la acción de $\mathrm{F}_{1}=10$.

En vista de que $\delta=\omega 2 / \omega 1=0.0001$ la masa $m_{2}$ está prácticamente desligada del sistema y sus desplazamientos $X_{2}$ son casi nulos del orden de $3 \times 10^{-4}$ para cualquier valor de $\gamma$ desde 0.1 hasta 2.0 , como se ve en la figura 2. Por lo que respecta a las masas $m_{1}$ y $m_{0}$ presentan los desplazamientos $X_{0}$ y $X_{1}$ mostrados en la figura 2 para $\gamma$ desde 0.1 hasta 2.0. La función de transferencia para $X_{1}$ presenta una gran amplificación de 5 para $\gamma=\omega / \omega 1=1$, esto es, cuando la frecuencia de excitación $\omega$ es igual a la frecuencia del sistema con masa $m_{1}$ y resorte $k_{1}$ de un grado de libertad. A bajas frecuencias el desplazamiento $X_{1}$ tiende al desplazamiento estático dado por el cociente $F_{1} / k_{1}=10 / 10=1$ y el desplazamiento de la masa $m_{0}$ es del orden de $1.125 \times 10^{-04}$. La frontera de un problema clásico modelada por $m_{0}$ tiene un desplazamiento infinitesimal, pero distinto de cero, lo que es necesario para mantener el sistema completo en equilibrio dinámico. La masa $m_{2}$ tiene desplazamientos del orden de $3 \times 10^{-04} \mathrm{en}$ vista de que es arrastrada por la fuerza viscosa $\xi_{2}=15 \%$ del amortiguamiento crítico.
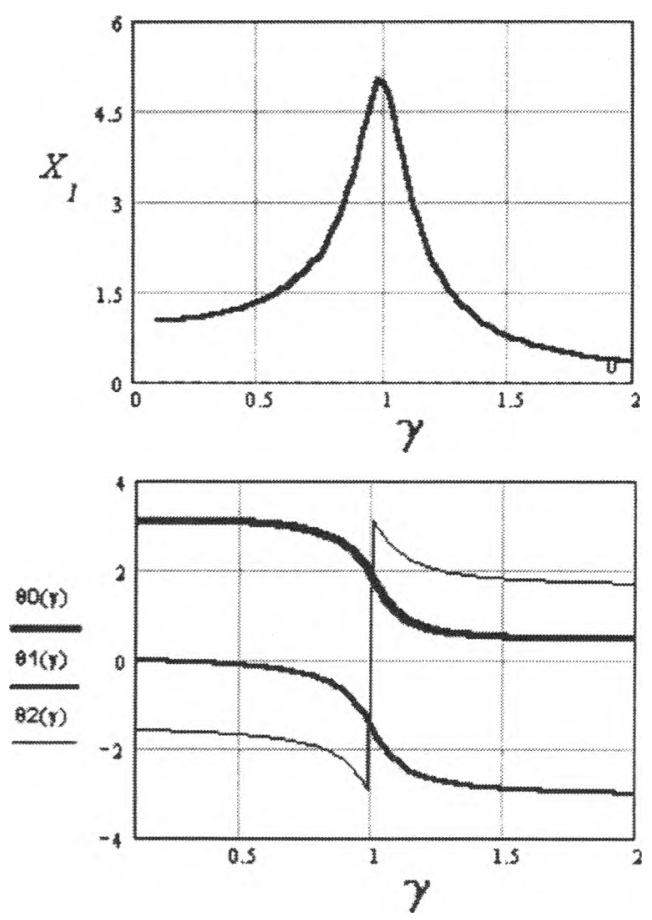
Si hubiéramos puesto un valor de $\xi_{2}=0.001 \%$ del amortiguamiento crítico, sería fácil ver desplazamientos que tendieran a cero muy rápidamente. Se hace notar que el sistema modelado en este problema necesita dos elementos (las masas $m_{0}$ y $m_{1}$ su resorte, así como su amortiguador) para el sistema dinámico más simple. Esto es así porque el nuevo modelo es más completo que el tradicional de un grado de libertad con sólo una masa $m_{1}$, que supone desplazamiento cero del apoyo, además no incluye la influencia de la inercia del apoyo proporcionado por la masa $m_{0}$ que modela al semiespacio de soporte.

\section{Análisis de una estructura con excitación en la base}

\section{Ejemplo 2}

El mismo sistema estructural adopta ahora los siguientes parámetros: resortes $k_{1}=k_{2}=10$, masa $m_{0}=1000000$ (unidades arbitrarias de masa), masas $m_{1}=m_{2}=10$ y amortiguamientos $\xi_{2}=$ $\xi_{2}=0.01$ esto es $1 \%$ del amortiguamiento crítico.

Supóngase que el terreno, modelado por la masa $m_{0}$, experimenta una aceleración unitaria $1^{*} e^{\mathrm{i} \omega t}$, en donde el parámetro adimensional que relaciona la frecuencia de excitación $\gamma=\omega / \omega_{1}$ variará de 0.1 a 10.0. Bajo estas circunstancias, el sistema completo será excitado por fuerzas $F_{1}$ y $F_{2}$ iguales a cero y una fuerza $F_{0}=1000000^{*} e^{\mathrm{i} \omega \mathrm{t}}$ (unidades de fuerza) que producirá una aceleración unitaria a la masa $m_{0}$, esto es $\ddot{u}_{g}=F_{0} / m_{0}=1^{*} e^{\mathrm{i} \omega t}$. Los resultados dados por las ecuaciones (9), (10) y (11) se muestran ahora en la figura 3.

Es claro que las frecuencias naturales de los componentes $m_{l}, k_{1} \mathrm{y} \mathrm{m}_{2}$ y $\mathrm{k}_{2}$ son $\omega_{1}=\omega_{2}=1$. Por tanto, si $\gamma=\omega / \omega_{1}$, entonces cuando $\gamma=0.1$ la frecuencia de excitación será $\omega=0.1$. El desplazamiento $m_{0}$ máximo a baja frecuencia debe se igual a $1 / \omega^{2}=1 /(0.1)^{2}=100$, como se indica en la figura 3(a) para $\gamma=0.1$. Esta es la prueba que garantiza la precisión de las ecuaciones (9), (10) y (11). Las masas $m_{1}$ y $m_{z}$ estando ligadas por resortes flexibles a la masa $m_{c}$ viajan un poquito más cuando $\gamma=0.1$ en distancias de 102.05 y 103.08 , respectivamente; esto está indicado en las figuras 3 (b) y (c). Otro resultado interesante se obtiene cuando se calculan y se grafican los desplazamientos relativos entre las tres masas del modelo. Estos resultados se muestran en la figura 4.

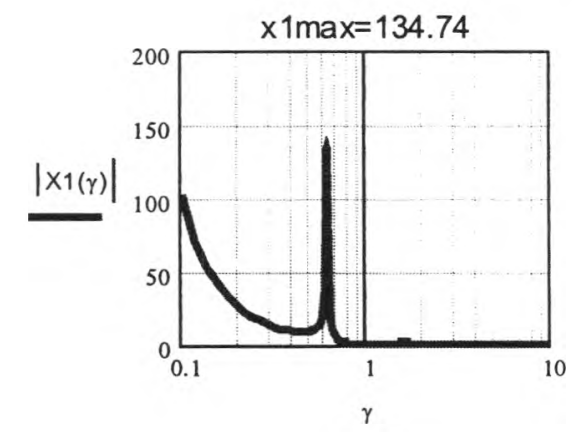

b)

a)

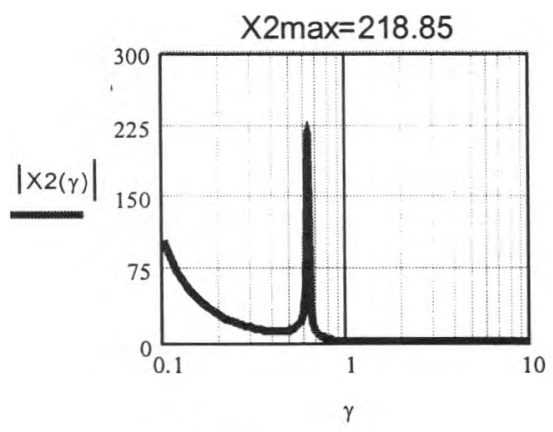

c)

Figura 3 


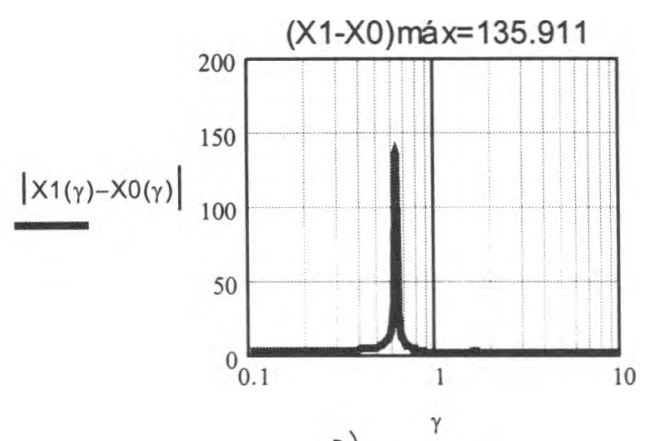

a)

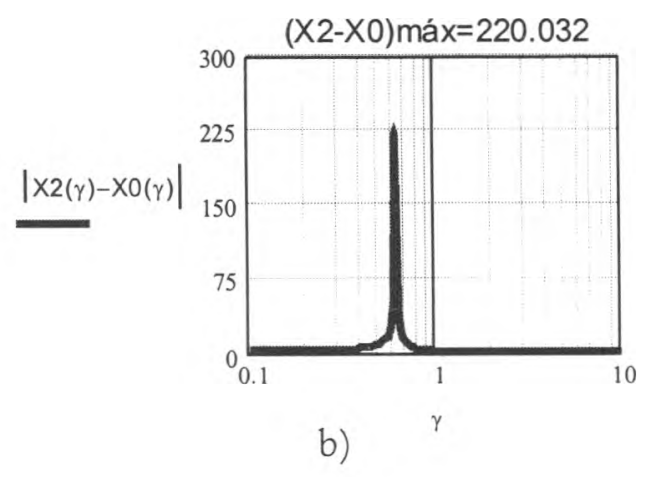

b)

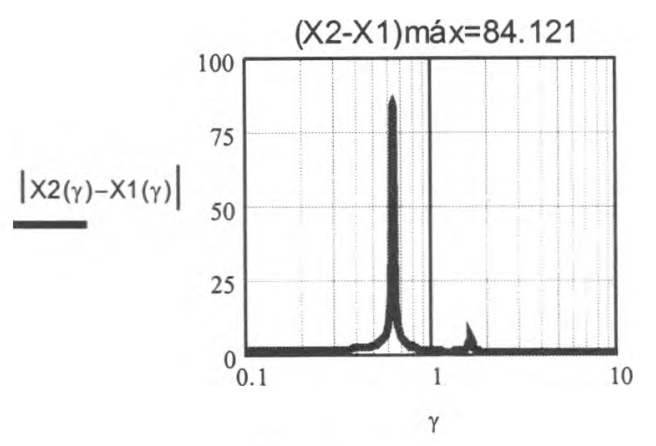

c)

Figura 4

En la parte superior de cada figura, el máximo desplazamiento relativo se indica con (Xi-Xj). Las frecuencias naturales del sistema completo ocurren aproximadamente para $\gamma=0.63$ y $1.70 \mathrm{y}$ debido a que $\omega_{1}=1$ entonces las frecuencias naturales del sistema son $\omega^{*}{ }_{1}$ y $\omega^{*}{ }_{2}$ son 0.63 y 1.70 $\mathrm{rad} / \mathrm{s}$.

Otro resultado importante que se puede obtener se muestra en la figura 5 de los ángulos de fase $\theta i(\gamma)$ para $i=0,1$ y 2 , con las tres masas vibrando (el cuadro más grande modela a $m_{0}$ ) de acuerdo a las diferencias de ángulos de fase de los desplazamientos. Una característica importante que se observa, es que el ángulo de fase $\theta 0(\gamma)$ de la masa $m_{0}$ siempre es constante $(-\pi)$ y siempre en fase con la excitación. En esta figura se puede ver que de $\gamma=0.1$ a 0.6 las tres masas viajan en fase y vibran como se indica debajo de la zona indicada. Para $\gamma=0.65$ a 0.95 las masas $m_{1}$ y $m_{2}$ vibran en fase y opuestas 180 grados con respecto a la masa $m_{0}$. En seguida para $\gamma=1.05$ a 1.65 las masas $m_{0} y m_{1}$ se ponen en fase y vibran en la misma dirección mientras que la masa $m_{2}$ vibra con una diferencia de fase de 180 grados, como se ve debajo de la región indicada. Finalmente, de $\gamma=1.75$ hasta $10 \mathrm{el}$ segundo modo es excitado como se indica. De estos análisis concluimos que cuando en un sistema estructural la excitación se hace por la base o frontera de una estructura dentro de cierto rango del parámetro $\gamma$ (en este caso de 0.1 a 10), todos y cada uno de los modos posibles de dicha estructura serán excitados. La importancia de este resultado se verá más adelante.

\section{Ejemplo 3}

Se analiza el mismo problema 2 con los siguientes parámetros: resortes $k_{1}=k_{2}=10$, masa $m_{0}=1000000$ (unidades arbitrarias de masa), masas $m_{1}=m_{2}=10$ y amortiguamientos $\xi_{2}=\xi_{2}=0.01$, esto es $1 \% \mathrm{del}$ amortiguamiento crítico. Supongamos de nuevo que la base modelada por la masa $m_{0}$ sufre una aceleración unitaria $1^{*} e^{\mathrm{i} \omega t}$, en donde la frecuencia de excitación $\omega$ varía nuevamente desde $\gamma=0.1$ hasta 10. En la literatura actual este problema de ingeniería sísmica se resuelve reemplazando las fuerzas generadas por el movimiento del terreno por fuerzas sísmicas equivalentes que son iguales en este caso a las masas $m_{1}$ y $m_{2}$ por la aceleración del terreno, denotada comúnmente por $\ddot{u}_{g}$. Bajo estas circunstancias el sistema será excitado por fuerzas $F_{1}$ y. $F_{2}$ iguales a 10 que 
producirán aceleración unitaria en las masas $m_{1} y$ $m_{2}$, esto es $\ddot{u}_{\mathrm{g}}=\mathrm{F}_{\mathrm{i}} / \mathrm{m}_{\mathrm{i}}=10 / 10=1$. La fuerza $F_{0}$ en la masa $m_{0}$ se hace igual a cero. El objetivo es ver las diferencias entre este modelo (el que actualmente se usa) y el modelo analizado en el ejemplo 2 de este artículo.

Los resultados para los desplazamientos dados por las ecuaciones (9), (10) y (11) se muestran en la figura 6.

En esta figura podemos observar los espectros de desplazamiento de las tres masas $m_{0}, m_{1}$ y $m_{2}$. Los máximos desplazamientos a baja frecuencia $\gamma=0.1$ para $X_{0}, X_{1}$ y $X_{2}$ son 0.002051 (unidades arbitrarias), 2.049 y 3.082 , respectivamente, y corresponden a la clásica configuración que se tendría si aplicáramos estáticamente las fuerzas $F_{1}=F_{2}=10$ a las masas $m_{1}$ y $m_{2}$. Las máximas amplificaciones ocurren a 0.64 $\mathrm{rad} / \mathrm{s}$, están indicadas en la parte superior de cada figura y son 0.0036 (tiende a cero, pero nunca es cero) para la masa $m_{0}, 135.91$ para la masa $m_{1}$ y 220.03 para la masa $m_{2}$. A la frecuencia $\omega=1.61 \mathrm{rad} / \mathrm{s}$ nuevamente ocurren amplificaciones de menor magnitud; sin embargo, los desplazamientos del semiespacio $m_{0}$ son del orden de $1.179 \times 10^{-5}$ y los de $m_{1}$ y $m_{2}$ del orden de -3.005 y 2.150 respectivamente (unidades de desplazamiento), indicando con esto la excitación del segundo modo. Esto último se puede observar en relación con el espectro de fases mostrado en la figura 7 , en donde se ilustran los ángulos de fase $\theta_{0}$ (línea gruesa) $\theta_{1}$ (línea media) y $\theta_{2}$ (línea delgada) de las masas $m_{0}, m_{1}$ y $m_{2}$, respectivamente.

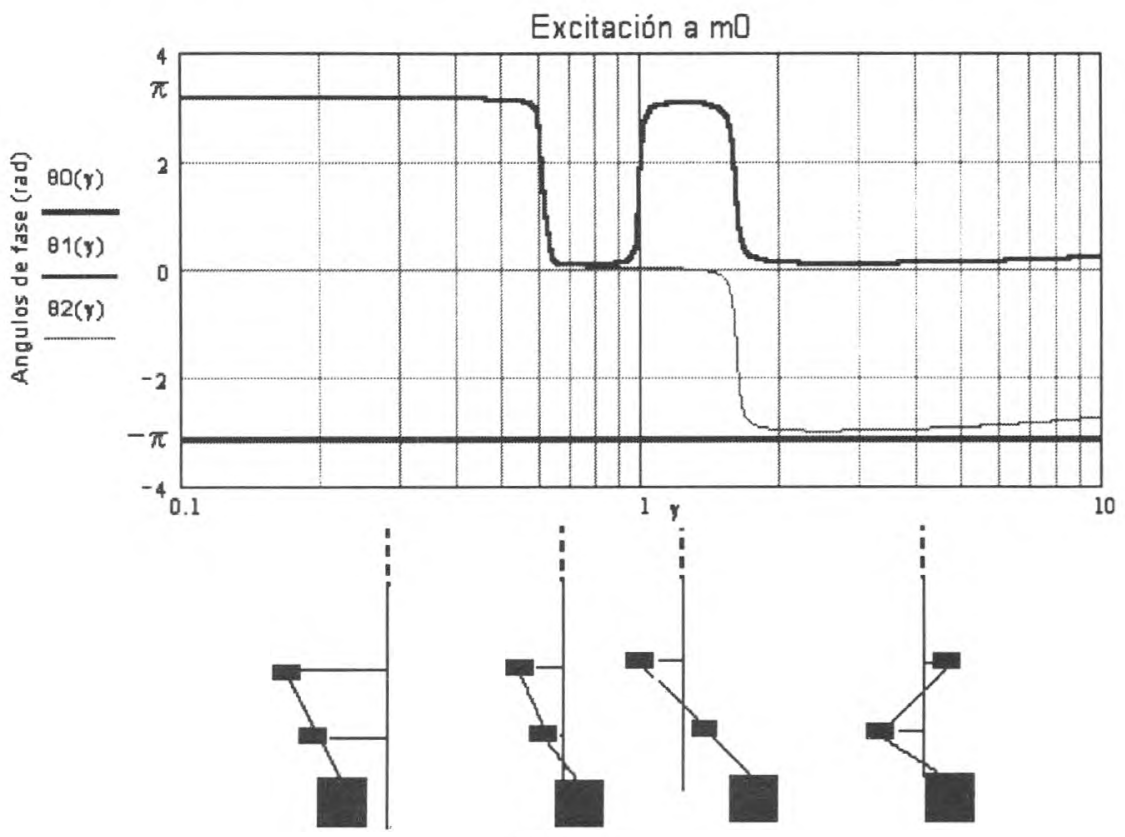

Figura 5

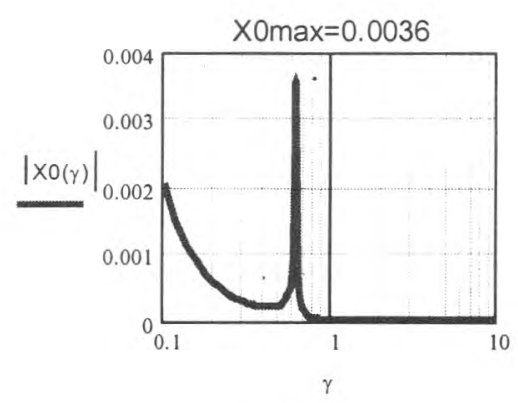

(a) $m_{0}$

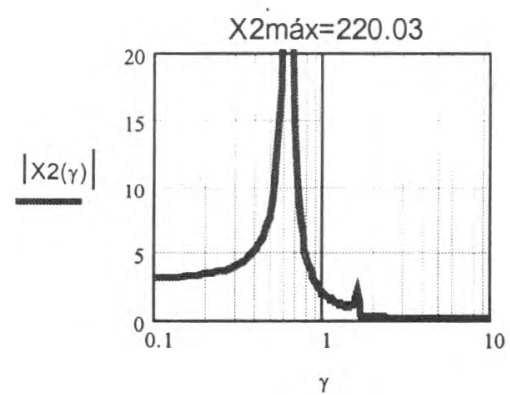

(b) $m_{1}$

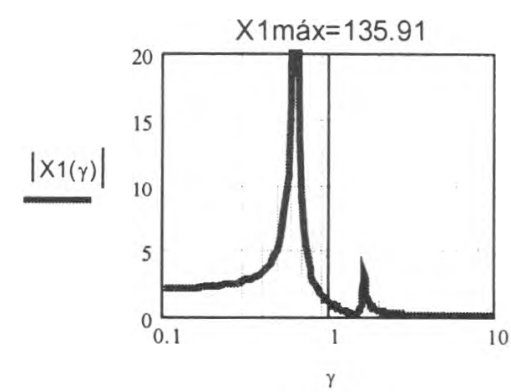

(c) $m_{1}$ 


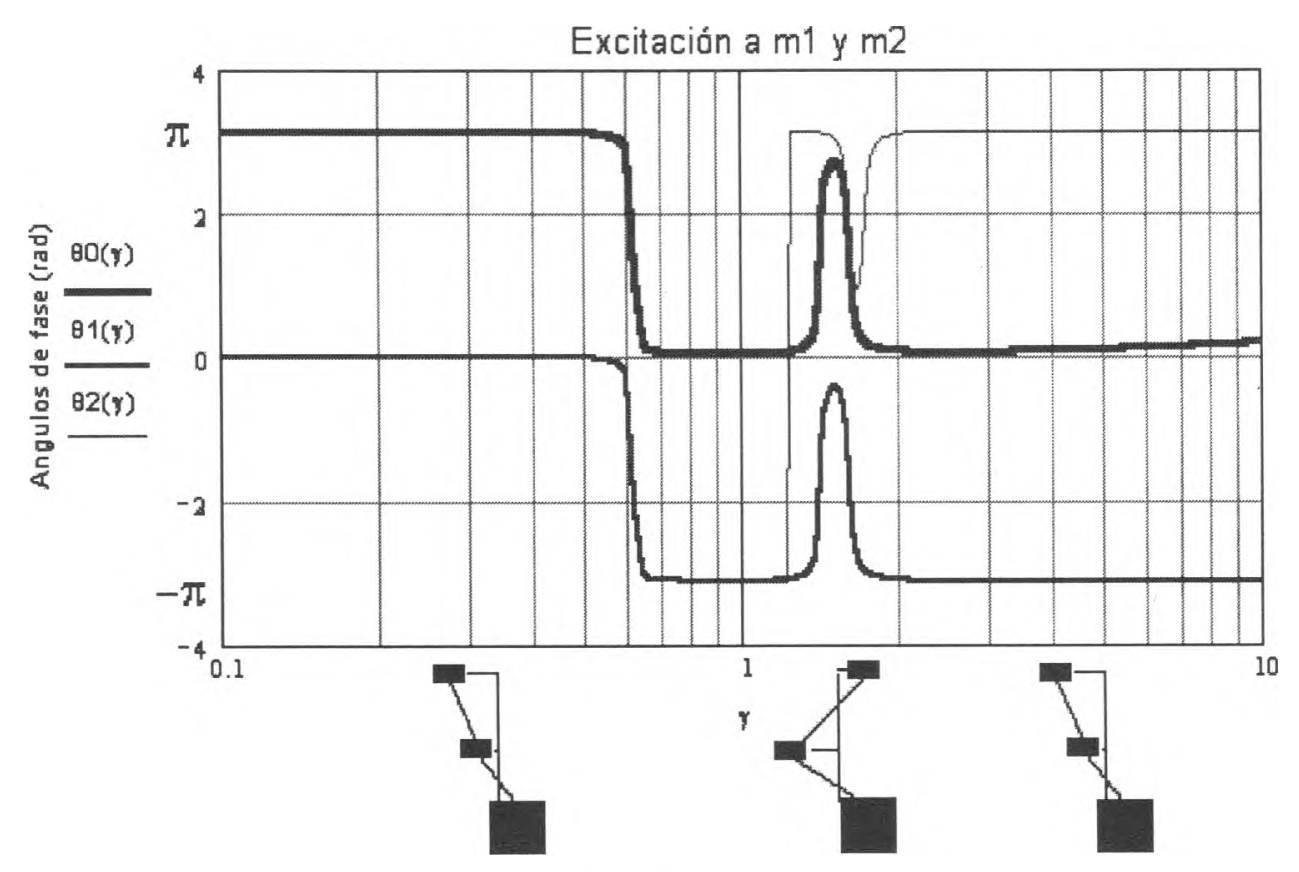

Figura 7

La característica más importante en la figura 7 es que aparte de la pequeña franja vertical de $1.52 \leq \gamma \leq 1.68$ en donde las masas $m_{0} y m_{2}$ van en fase $\left(\theta_{0}\right.$ y $\theta_{2}$ prácticamente se traslapan) en el resto del espectro de fase de 0.1 a 1.52 y de 1.68 a 10.0 el modo que siempre se excita es el clásico primer modo con el semiespacio $m_{0}$ vibrando en sentido opuesto a los desplazamientos efectuados por las masas $m_{1}$ y $m_{2}$. Obsérvese cómo $\theta_{1}$ y $\theta_{2}$ siempre están aproximadamente 180 grados $( \pm \pi)$ fuera de fase con respecto a $m_{0}$.

Ahora podemos concluir que el método de usar fuerzas sísmicas efectivas iguales a las masas $m_{i}$ multiplicadas por la aceleración del terreno $\ddot{u}_{g}$ para el problema de movimiento en la base (sin ninguna restricción) no aproxima más que la respuesta del primer modo clásico de vibración. Se puede ver en la figura 5 que en el análisis de excitación inducida por la base, todos y cada uno de los modos son subsecuentemente excitados dentro del rango de frecuencias que contenga a las frecuencias del sistema. Esto puede ser de notorias consecuencias en el caso de estructuras altas y flexibles con frecuencias naturales poco espaciadas (alta densidad de frecuencias).
Para terminar, graficamos la diferencia de desplazamientos entre cada par de masas del sistema, en donde encontramos que las gráficas son idénticas a aquéllas mostradas en la figura 4 hasta el último dígito. La conclusión de esto es que la diferencia $\left|X_{i}(\gamma)-X_{j}(\gamma)\right|$ para $\mathrm{i}=0,1$ y 2 con $\mathrm{i} \neq \mathrm{j}$ es una cantidad tensorial invariante bajo las hipótesis y condiciones de carga asumidas en los ejemplos 2 y 3 .

\section{Conclusiones}

La principal característica del problema de excitación inducida por la frontera (Figura 5), es que todos los modos son excitados de manera consecutiva al incrementar la frecuencia de excitación. Este hecho va en contraste con el método a proximado de reemplazar las fuerzas de un sismo por fuerzas sísmicas efectivas. Cuando usamos fuerzas efectivas $m_{i} \ddot{u}_{g}$, estas excitaciones van siempre en fase y en sentido contrario a los movimientos efectuados por la base, excepto, muy localmente, en torno a la frecuencia natural del primer modo clásico de vibrar (Figura 7). Sin embargo, hacemos notar que se pueden usarlas clásicas fuerzas efectivas, para un análisis aproximado de las fuerzas sísmicas de diseño, causadas por el efecto del primer modo, y para ello, sólo escogemos una pequeña región del espectro del 


\section{Referencias}

sismo elegido y que esté centrada con respecto a la primera frecuencia natural de la estructura que se estudie. El uso del resto del espectro del sismo de diseño nos proporcionará bajo estas circunstancias, contribuciones inciertas del primer modo de vibración. Finalmente, el lector observará que cuando al modelo se le impone alguna excitación a la primera masa $m_{0}$, pero las masas $m_{1}$ y $m_{2}$ son del mismo orden que la masa $m_{0}$, entonces estaremos modelando una máquina de ferrocarril que arrastra dos vagones (o algún robot caminando), estoes, podemos modelar ya una estructura con todas las masas libres de moverse. En un artículo posterior, enfocaremos con más detalle el análisis de alguna estructura en movimiento.
Bettin I., Urrutia-Galicia J.L. y Gebbeken N. (2000). On the Análisis of Vertical Circular Cylindrical Tanks under Earthquake Excitation at its Base.Universität der Bundeswehr München, UniBw-BKI 00/2000, Deutschland (Alemania). Thomson W.T. (1972). Theory of Vibration with Applications. Prentice Hall, Inc. N.J.

Tse F.S., Morse I.E. y Hinkle R.T. (1978). Mechanical Vibrations, Theory and Applications. Allyn and Bacon, Inc.

Urrutia-Galicia J.L. (1998). On the Absolute Form of the Theory of Dynamics for Beams, Plates and Shells with Applications for Engineers. Algebra and Functional Analysis. Mitteilungen des Institutes für Statik der Universität Hannover, Mitteilung Nr. 47-98, Hannover, Deutschland (Alemania).

\section{Semblanza del autor}

José Luis Urrutia-Galicia. Obtuvo el grado de ingeniero civil en la Facultad de Ingeniería de la UNAM en 1975; asimismo, los grados de maestría (1979) y doctorado (1984) en la Universidad de Waterloo, en Ontario, Canadá. Es investigador del Instituto de Ingeniería, UNAM en la Coordinación de Mecánica Aplicada. Sus áreas de interés cubren: matemáticas aplicadas y mecánica teórica, análisis tensorial, estabilidad y vibraciones de sistemas discretos, vigas, placas y cascarones. Ha recibido reconocimientos como el "Premio al Mejor Artículo" de las Transacciones Canadienses de Ingeniería Mecánica (CSME) (Montreal, Canadá 1987) por el artículo "The Stability of Fluid Filled, Circular Cylindrical Pipes, part II Experimental", también le fue otorgada la "Medalla Duggan", que es la más alta distinción otorgada por la CSME (en la Universidad de Toronto, Canadá, 1990) por el artículo "On the Natural Frequencies of Thin Simply Supported Cylindrical Shells. 